\title{
Dermatologically relevant biomedicinal plants in Manipur, India
}

\section{Bishurul Hafi ${ }^{1}$, Nandakishore Singh ${ }^{2}$, Bimola Devi ${ }^{3}$, Khumukcham Nongalleima ${ }^{4}$, Sanalembi Mutum², Romita Bachaspatimayum²}

${ }^{1}$ Department of Dermatology, Venereology and Leprology, IQRAA International Hospital and Research Institute, Kozhikkod, Kerala, India, ${ }^{2}$ Department of Dermatology, Venereology and Leprology, Regional Institute of Medical Sciences, Imphal, Manipur, India, ${ }^{3}$ Department of Chemistry, Imphal college, Manipur, India, ${ }^{4}$ Department of Biotechnolog, Institute of Bioresource and Sustainable Development, Imphal, India

Corresponding author: Dr. Bishurul Hafi, E-mail: bishuru@gmail.com

\begin{abstract}
Background: Manipur is a border state of north-eastern India and is a part of Indo-Myanmar biodiversity hot spot. The current study was done to assess the different flora commonly used by the locals for the dermatological issues. Methodology: All the available articles published between 2013 December to 2016 June were searched in 2 search engines (Pubmed and Google scholar) as well as 4 scientific journals (Indian Journal of Traditional Knowledge, Journal of ethnopharmacology, Journal of Economic and Taxonomic Botany and Indian Journal of natural products) with keywords biomedicinal plants, Manipur and skin disorders. A total of 459 articles were analysed and only 19 articles were found relevant to the subject. All the plant and plant products traditionally used for skin infections were tabulated. It was cross checked by research scholars in Institute of Bio resource and Human Development, Imphal. Further, their usage was confirmed with local traditional healers. Results: 31 plants were seen used as antifungal agents, 21 antibacterial agents, 16 anti scabitic agents, 6 anti pruritic medicines, 4 plants used to treat leukoderma, 7 anti acne agents, 4 products to use in eczema, 5 anti leprotic agents, 17 plant products to enhance wound healing with 6 plants specialised for burn wounds, 5 anti gonorrhoeal plants, 3 plants used in oral ulcers and 12 hair supplements. But none of the plants were studied scientifically. Conclusion: True clinical trials as well as scientific laboratory based examinations to decipher the contents of the herbs were not carried out effectively yet.
\end{abstract}

Key words: Medicinal plants; Traditional medicine; Northeast

\section{INTRODUCTION}

Manipur is a border state of north-eastern India situated between $23.83^{\circ} \mathrm{N}$ and $25.68^{\circ} \mathrm{N}$ latitude and $93.03 \mathrm{oE}$ and $94.78 \mathrm{oE}$ longitude. It shares border with Myanmar and is a part of Indo-Myanmar biodiversity hot spot. It comprises $1820 \mathrm{sq} . \mathrm{km}$ of flat valley surrounded by 20507sq.km of hill territory and forms a part of the Himalayan mountain system. Meitei (Manipuri) are the majority community who along with Meitei pangal (Manipuri Muslim) inhabit the valley region whereas the hilly areas are inhabited by 30 different tribes. Manipur is known for its ecologically distinctive and rich biodiversity, having forest in $60 \%$ of its total land.
More importantly most of the traditional knowledge are still preserved and actively used in daily life for healing in natural ways. The current study was done to assess the different flora commonly used by locals for the dermatological problems.

\section{METHODOLOGY}

All the available articles published between 2013 December to 2016 June were searched in two search engines (Pubmedand Google scholar) as well as four scientific journals (Indian Journal of Traditional Knowledge, Journal of Ethnopharmacology, Journal of Economic and Taxonomic Botany and Indian Journal

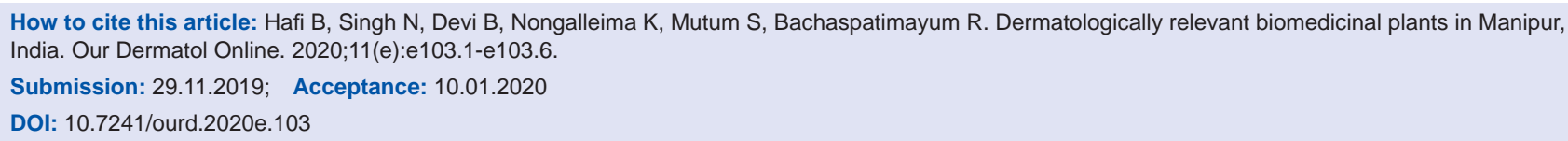


of natural products) with keywords biomedicinal plants, Manipur and skin disorders. A total of 459 articles were analysed and only 19 articles were found relevant to the subject. All the plant and plant products traditionally used for different dermatological conditions were tabulated. It was cross checked by research scholars in the Institute of Bioresources and Human Development, Imphal. Further, their usage was confirmed with local traditional healers (maibas and maibis) by open end interview technique.

\section{RESULTS}

We could find out 31 plants used as antifungal agents, 21 antibacterial agents, 16 anti scabitic agents, 5 anti leprotic agents, 5 anti gonorrhoeal plants (Table 1), 6 anti pruritic medicines (Table 2), 4 plants used to treat leukoderma (Table 3), 7 anti acne agents (Table 4), 4 products to use in eczema, 1 antipsoriatic agent (Table 5), 17 plant products to enhance wound healing with 6 plants specialised for burn wounds, 3 plants used in oral ulcers (Table 6), 1 as emollient, 12 hair supplements and 35 plants extracts used to prepare natural herbal shampoo (Table 7).

\section{DISCUSSION}

$65-80 \%$ of world's population usecomplementaryand alternative medicines (CAM) as treatment modality. Prevalence is higher among developing countries and rising in developed ones [1]. These medicinal systems are heavily dependent on various plant species and plant based products. Some species are endemic and are becoming increasingly rare and at the verge of extinction. Hundreds of plant products were in use from time immemorial to treat dermatological conditions (Figs. 1 - 2).

In a detailed study among Lois tribes Andro village, 42 plant species were found to be actively used for skin disorders. The 42 plant species belonged to 39 genera which are distributed over 22 families. Plant parts used and mode of usage has been described in detail [1].

Inaocha et al has tabulated the collective list of wild biomedicinal plants used in tribes of the hills of Manipur. The investigation reported 100 species of 41 families with 63 aromatic and 37 non-aromatic plants. They traced five critically endangered plants, still growing wild and over exploited for medicinal and commercial purposes [4].

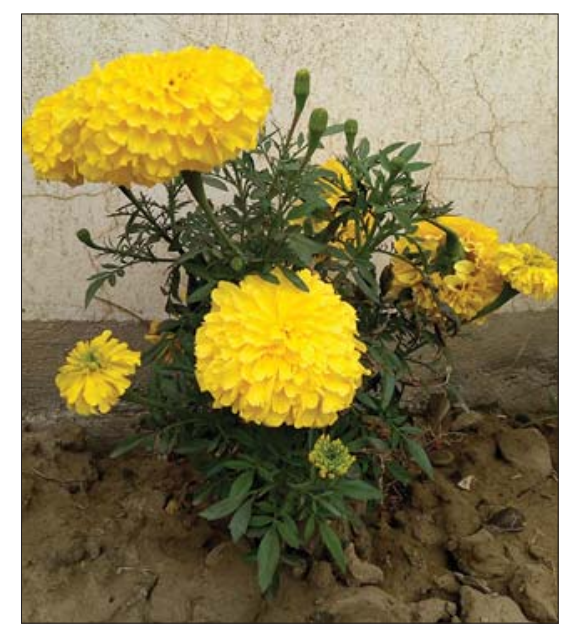

Figure 1: Scientific name- Tegeteserecta (Asteraceae), Vernacular name- Sanareiathonba, leaves are used in herbal shampoo preparation.

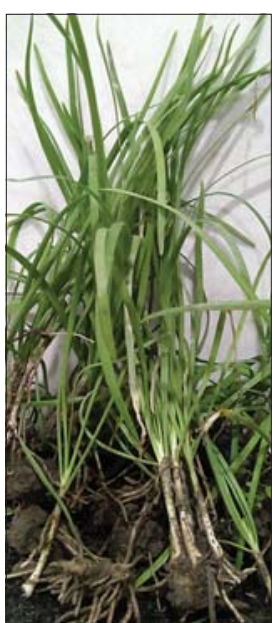

Figure 2: Scientific name- Allium odorum (Liliaceae), vernacular name- Maroinakupi, whole plant is used as antibacterial agent.

Another study by Leishangthem et al described medicinal values of 50 plants commonly seen in Imphal East district. Out of it nine are widely used for dermatological conditions. It was a questionnaire based study conducted in different remote villages [5].

Study by Rita $\mathrm{N}$ et al explained an ancient cosmetic and strengthening method of tooth among Maring tribes. They used fruit of Garcinia pedunculata, locally known as Heirou, was cut into thin slices and roasted in fire. Some small reddish black coloured fruit of Melastomamalabathricum, locally known as Yachubi was also roasted. Both the roasted fruit are taken orally and kept inside the mouth avoiding swallowing of the same and at the same time the teeth are exposed to the heat of burning charcoal. This process of exposure to heat is allowed for few minutes until the teeth turns into blackish colour [6]. 
Table 1: Plants used in infections [1-10]

\begin{tabular}{|c|c|c|}
\hline Scientific name (Family) & $\begin{array}{l}\text { Vernacular } \\
\text { name }\end{array}$ & Parts used \\
\hline \multicolumn{3}{|l|}{ Antifungal } \\
\hline Juglans regia (Juglandaceae) & Heijuga/Heijugak & Leaves \\
\hline $\begin{array}{l}\text { Lithocarpus elegans } \\
\text { (Fagaceae) }\end{array}$ & Kuhi & Stem bark \\
\hline $\begin{array}{l}\text { Alpinia galangal } \\
\text { (Zingiberaceae) }\end{array}$ & Kanghoo & Rhizome \\
\hline $\begin{array}{l}\text { Nicotiana plumbaginifolia } \\
\text { (Solanaceae) }\end{array}$ & Meitei hidak mana & Leaves \\
\hline Capsicam annum (Solonaceae) & Meitei Morok & Leaves \\
\hline $\begin{array}{l}\text { Drymariacordata } \\
\text { (Caryophyllaceae) }\end{array}$ & Tandanpambi & Whole plant \\
\hline $\begin{array}{l}\text { Ipmoeaacqatica } \\
\text { (Convolvulaceae) }\end{array}$ & Kolamni & Whole plant \\
\hline Bombax ceiba & Tera & - \\
\hline Cassia fistula & Chahui & - \\
\hline $\begin{array}{l}\text { Acacia catechu } \\
\text { (Febacea or Leguminocaceae) }\end{array}$ & Katha & $\begin{array}{l}\text { Seed, tender, } \\
\text { pod }\end{array}$ \\
\hline $\begin{array}{l}\text { Curcurma angustifolia } \\
\text { (Zingiberaceae) }\end{array}$ & Yaipal & Inflorescence \\
\hline Mikania scandens (Asteraceae) & Uri hingchabi & Whole plant \\
\hline Lantana lamara (Verbenaceae) & Nongbaslei & Leaves, fruit \\
\hline $\begin{array}{l}\text { Plectranthusternifolius } \\
\text { (Lamiaceae) }\end{array}$ & Khoiju & Leaves \\
\hline Toona ciliate (Maliaceae) & Tairel & Leaves \\
\hline Vitex trifolia (Verbenaceae) & Urik-shibi & Leaves \\
\hline $\begin{array}{l}\text { Calotropis gigantean } \\
\text { (Asclepiadaceae) }\end{array}$ & Ang-got & Shoot \\
\hline Pistia stratiotes & Kangjao & Whole plant \\
\hline Ocimum sanctum (Lamiaceae) & Lurimi & Leaves \\
\hline $\begin{array}{l}\text { Alpinia galanga } \\
\text { (Zingiberaceae) }\end{array}$ & Aital & Rhizome \\
\hline $\begin{array}{l}\text { Pandanus odoratissimus } \\
\text { (Pandanaceae) }\end{array}$ & Gamlengthei & Flower \\
\hline $\begin{array}{l}\text { Rumexmaritimus } \\
\text { (Polygonaceae) }\end{array}$ & Torongkhongchak & Leaves \\
\hline Brassica juncea (Brassicaceae) & Hangam & - \\
\hline Brassica rapa (Brassicaceae) & Salgam & - \\
\hline Croton tiglium (Euphorbiaceae) & Koni-bih & - \\
\hline Helianthus annus (Asteraceae) & Numitlei & - \\
\hline $\begin{array}{l}\text { Madhucalongifolia } \\
\text { (Sapotaceae) }\end{array}$ & Nageshor & - \\
\hline Olea europa (Oleaceae) & Chorfon & - \\
\hline $\begin{array}{l}\text { Ricinus communis } \\
\text { (Euphorbiaceae) }\end{array}$ & Kege & - \\
\hline $\begin{array}{l}\text { Capsellabursapastoris } \\
\text { (Brassicaceae) }\end{array}$ & Chantruk & - \\
\hline \multicolumn{3}{|l|}{ Antibacterial } \\
\hline $\begin{array}{l}\text { Jatropha curcas } \\
\text { (Euphorbiaceae) }\end{array}$ & Awa kege & Latex, seed \\
\hline $\begin{array}{l}\text { Bauhinic acuminate } \\
\text { (Leguminosae) }\end{array}$ & Chingthraoangonba & Stem bark \\
\hline Bombax ceiba (Malvaceae) & Tera & - \\
\hline Cassia fistula (Fabaceae) & Chahui & - \\
\hline $\begin{array}{l}\text { Oroxylumindicum } \\
\text { (Bignoniaceae) }\end{array}$ & Shamba & - \\
\hline $\begin{array}{l}\text { Catharanthus roseus } \\
\text { (Apocynaceae) }\end{array}$ & Saheb lei & - \\
\hline $\begin{array}{l}\text { Drymariacordata } \\
\text { (Caryophyllaceae) }\end{array}$ & Tandanpambi & - \\
\hline $\begin{array}{l}\text { Zingibercapitatum } \\
\text { (Zingiberaceae) }\end{array}$ & Lam-shing & Rhizome \\
\hline
\end{tabular}

Table 1: (Continued)

\begin{tabular}{|c|c|c|}
\hline Scientific name (Family) & $\begin{array}{l}\text { Vernacular } \\
\text { name }\end{array}$ & Parts used \\
\hline $\begin{array}{l}\text { Dryopteris marginata } \\
\text { (Polypoidiaceae) }\end{array}$ & Lai-chanchrang & Leaves \\
\hline $\begin{array}{l}\text { Curcurma angustifolia } \\
\text { (Zingiberaceae) }\end{array}$ & Yaipal & Inflorescence \\
\hline $\begin{array}{l}\text { Xylosmalongifolia } \\
\text { (Flacourtiaceae) }\end{array}$ & Nong-leishang & Leaves, fruit \\
\hline Pinus kesiya (Pinaceae) & Uchan & Wood, leaves \\
\hline Toona ciliate (Meliaceae) & Tairel & Leaves \\
\hline Allium ascalonium (Liliaceae) & Tilhou-macha & Leaves, bark \\
\hline Elsholtzia ciliate (Lamiaceae) & Tekta & Leaves \\
\hline Allium odorum (Liliaceae) & Maroinakupi & Whole plant \\
\hline Allium hookeri (Alliaceae) & Maroinapakpi & Whole plant \\
\hline $\begin{array}{l}\text { Argyreia nervosa } \\
\text { (Convolulaceae) }\end{array}$ & Poongdinglee & Leaves \\
\hline $\begin{array}{l}\text { Phologacanthusthyriformis } \\
\text { (Acanthaceae) }\end{array}$ & Nongmangkha & Shoot, rhizome \\
\hline Juglans regia (Juglandaceae) & Heijugak & \\
\hline Anesomitesindica (Lamiaceae) & Thoidingangouba & \\
\hline \multicolumn{3}{|l|}{ Anti-scabitic } \\
\hline $\begin{array}{l}\text { Jatropha curcas } \\
\text { (Euphorbiaceae) }\end{array}$ & Awa kege & Latex, seed \\
\hline $\begin{array}{l}\text { Lithocarpus elegans } \\
\text { (Fagaceae) }\end{array}$ & Kuhi & Stem bark \\
\hline Arundo donax (Poaceae) & Yengthou & Tender shoots \\
\hline Mimosa pudica (Mimosaceae) & Kangaphalekaithabi & Whole plant \\
\hline Azadirachtaindica (Meliaceae) & $\mathrm{Nim}$ & Leaves \\
\hline $\begin{array}{l}\text { Bauhinia acuminata } \\
\text { (Leguminosae) }\end{array}$ & Chingthraoangouba & Stem bark \\
\hline Capparis tenera (Capparaceae) & Kakyelkhujin & Leaves \\
\hline Curcuma longa (Zingiberaceae) & Yaingang & Rhizome \\
\hline $\begin{array}{l}\text { Drymariacordata } \\
\text { (Caryophyllaceae) }\end{array}$ & Tandanpambi & Whole plant \\
\hline $\begin{array}{l}\text { Buddlejaasiatica } \\
\text { (Buddlejacaceae) }\end{array}$ & Ngamurei & - \\
\hline $\begin{array}{l}\text { Gossypium arboretum } \\
\text { (Malvaceae) }\end{array}$ & Chaning & Flower \\
\hline $\begin{array}{l}\text { Manihot esculenta } \\
\text { (Euphorbiaceae) }\end{array}$ & U-mangra & Leaves \\
\hline Nerium indicum (Apcynaceae) & Kabireiangangba & Leaves \\
\hline Tagetespatula (Asteraceae) & Hao-sanarei & Leaves \\
\hline Toonaciliata (Meliaceae) & Tairen & Leaves \\
\hline Vitex trifolia (Lamiaceae) & Urikshibi & Whole plant \\
\hline \multicolumn{3}{|l|}{ Anti-leprosy } \\
\hline Azadirachtaindica (Meliaceae) & $\mathrm{Nim}$ & Leaves \\
\hline $\begin{array}{l}\text { Calotropis gigantean } \\
\text { (Asclepindacea) }\end{array}$ & Ang-got & Shoot \\
\hline $\begin{array}{l}\text { Jatropha gossypifolia } \\
\text { (Euphorbiaceae) }\end{array}$ & E-hidak & Leaves, shoot \\
\hline $\begin{array}{l}\text { Zingiber zerumbet } \\
\text { (Zingiberaceae) }\end{array}$ & Singkha & Rhizome \\
\hline $\begin{array}{l}\text { Dioscoreaalata } \\
\text { (Dioscoreaceae) }\end{array}$ & Hakaisan & Tuber \\
\hline \multicolumn{3}{|l|}{ For Gonorrhea } \\
\hline $\begin{array}{l}\text { Phyllanthus acidus } \\
\text { (Phyllanthaceae) }\end{array}$ & Gihori & - \\
\hline $\begin{array}{l}\text { Equisetum debile } \\
\text { (equisetaceae) }\end{array}$ & Lai-utong & Leaves \\
\hline Houttuynia cordata (Sauraceae) & Toningkhok & Leaves, rhizome \\
\hline $\begin{array}{l}\text { Magnolia champaca } \\
\text { (Magnoliaceae) }\end{array}$ & Leihao & $\begin{array}{l}\text { Inflorescence, } \\
\text { root }\end{array}$ \\
\hline Portulacaoleraceae & Leipakkundo & Shoot \\
\hline
\end{tabular}


Table 2: Plants used for pruritus $[1,3,4,6]$

\begin{tabular}{|c|c|c|}
\hline Scientific name (Family) & Vernacular name & Parts used \\
\hline $\begin{array}{l}\text { Bauhinia acuminata } \\
\text { (Leguminosae) }\end{array}$ & Chingthraoangonba & Stem bark \\
\hline $\begin{array}{l}\text { Capparis tenera } \\
\text { (Capparaceae) }\end{array}$ & Kakyelkhujin & Leaves \\
\hline $\begin{array}{l}\text { Chenopodium Albans } \\
\text { (Amaranthaceae) }\end{array}$ & Monsaobi & - \\
\hline $\begin{array}{l}\text { Acorus calamus } \\
\text { (Acaceae) }\end{array}$ & Oak-hidak & $\begin{array}{l}\text { Leaves, root, } \\
\text { rhizome }\end{array}$ \\
\hline $\begin{array}{l}\text { Sagattariasagittifolia } \\
\text { (Alismataceae) }\end{array}$ & Koukha & Leaves \\
\hline $\begin{array}{l}\text { Curcuma amada } \\
\text { (Zingiberaceae) }\end{array}$ & Yaiheinouman & Whole plant \\
\hline
\end{tabular}

Table 3: Plants used for leukoderma $[3,7,10]$

\begin{tabular}{lll}
\hline Scientific name (Family) & Vernacular name & Parts used \\
\hline Calotropis gigantean (Apocynaceae) & Angkot & Latex \\
Mussaendra glabra (Rubiaceae) & Hanurei & Roots \\
Alpinia galanga (Zingiberaceae) & Kaang hu & rhizome \\
Cassia fistula (Fabaceae) & Chahui & - \\
\hline
\end{tabular}

Table 4: Plants used for acnie $[1,2,7,10]$

\begin{tabular}{lll}
\hline Scientific name (Family) & Vernacular name & Parts used \\
\hline Mimosa pudica (Mimosaceae) & Kangphalekaithabi & Whole plant \\
Ipomoea aquatica (Convolvulaceae) & Kolamni & Whole plant \\
Bombex ceiba (Bombacaceae) & Tera & - \\
Caprica papaya (Caricaceae) & Awathabi & - \\
Lagenariasicereria (Cucurbitaceae) & Khongdrum & Fruit \\
Musa paradisiaca (Musaceae) & Laffutharo & Fruit \\
Plantagoerosa (Plantaginaceae) & Yempat & Leaves, fruits \\
\hline
\end{tabular}

Ranibaladevi et al listed 32 plant species belonging to 25 families used for dermatological issues among Paite tribe of Manipur. Information was collected by interviewing local traditional healers and the modes of usage with different plants are effectively addressed [7].

Meitei (Manipuri) community inhabiting in the valley regions have the traditional knowledge of using natural herbal shampoo called "Chinghi" from non historical time itself to treat different ailments of hair like antiageing of the hair, black and shininess of the hair [12]. It is prepared from the local rice water 'Chinghi' along with many herbs. In this rice water, natural herbs and leaves of fruit trees as mentioned in the Table 1 are added and boiled properly. After the ingredients are properly boiled, it is cooled down and sieved using a muslin cloth to remove the impurities and the finally collected clear liquid is used as herbal shampoo. After using the herbal shampoo for washing the hair, the hair is washed properly and no oil is needed to apply on the hair afterwards. It is best to use it within 2-3 days after preparation. The fermented lime is also used as natural herbal shampoo by the Meitei community particularly as anti-ageing for the hair preventing grayness of the
Table 5: Plants used for papulosquamous disorders [1,3,5,7]

\begin{tabular}{|c|c|c|}
\hline $\begin{array}{l}\text { Scientific name } \\
\text { (Family) }\end{array}$ & $\begin{array}{l}\text { Vernacular } \\
\text { name }\end{array}$ & $\begin{array}{l}\text { Parts } \\
\text { used }\end{array}$ \\
\hline \multicolumn{3}{|l|}{ For eczema } \\
\hline Setariapumila (Poaceae) & Hup & Whole plant \\
\hline Jatropha gossypifolia (Euphorbiaceae) & E-hidak & Leaves, root \\
\hline Glycosmis pentaphylla (Rutaceae) & Yong komla & Leaves \\
\hline Tagetespatula (Asteraceae) & Hao-sanarei & Leaves \\
\hline \multicolumn{3}{|l|}{ For psoriasis } \\
\hline Phyllanthus acidus (Phyllanthaceae) & Gihori & - \\
\hline
\end{tabular}

Table 6: Plants used for wound care $[3-7,10,11]$

\begin{tabular}{|c|c|c|}
\hline Scientific name (Family) & $\begin{array}{l}\text { Vernacular } \\
\text { name }\end{array}$ & $\begin{array}{l}\text { Parts } \\
\text { used }\end{array}$ \\
\hline \multicolumn{3}{|l|}{ For wound healing } \\
\hline Sonchus asper (Asteraceae) & Khom-thokpi & Leaves \\
\hline Cynodondactylon (Poaceae) & Tingthau & Leaves \\
\hline Eucalyptus citriodora (Myrataceae) & Nasik & Leaves \\
\hline Gynuracusimba (Asteraceae) & Terapaibi & leaves \\
\hline Pogostemonbengalensis (Lamiaceae) & Lamthoiding & Leaves, root \\
\hline Pogostemonparviflavus (Asteraceae) & Sangbrei & Whole plant \\
\hline Adenostemmalavenia (Asteraceae) & Lalu-kok & Leaves \\
\hline Cymbopogon flexosus (Gramineae) & Haona & Leaves \\
\hline Commelinadiffusa (Commenlinaceae) & Wandengkhoibi & - \\
\hline Curcuma domestica (Zingiberaceae) & Yai-ngang & Rhizome \\
\hline $\begin{array}{l}\text { Crassocephalumcrepidiodes } \\
\text { (Asteraceae) }\end{array}$ & Tera paibi & - \\
\hline \multicolumn{3}{|l|}{ For burns } \\
\hline Zingibercapitatum (Zingiberaceae) & Lam-shing & Rhizome \\
\hline Dryopteris marginata (Polypodiaceae) & Lai-chankharang & Leaves \\
\hline Rumexmaritinus (Polygonaceae) & Torong-khongchak & Whole plant \\
\hline Artocarpusheterophyllus (Moraceae) & Theibong & - \\
\hline Luffa cylindrica (Cucurbitaceae) & Sebot & Leaves \\
\hline $\begin{array}{l}\text { Phologacanthusthyriformis } \\
\text { (Acanthaceae) }\end{array}$ & Nongmangkha & - \\
\hline \multicolumn{3}{|l|}{ For aphthous ulcer } \\
\hline $\begin{array}{l}\text { Euphorbia euterophylla } \\
\text { (Euphorbiaceae) }\end{array}$ & Pakhang-leiton & Whole plant \\
\hline Neptumia oleracea (Fabaceae) & Eshingekaithopi & Leaves \\
\hline Portulacaoleraceae (Portulacaceae) & Leipakkundo & Shoot \\
\hline
\end{tabular}

hair. It is prepared from the ripe lime locally called "Champra". First, it is washed properly with the water then the juice is extracted after removing the seeds. Then the fruit along with the peel is cut into small pieces and kept in air tight plastic bottle or glass bottle under the room temperature in dark place. The fermentation process is completed within 2 weeks but the bottle should not be opened until it is completely fermented. After the fermentation is completed, it is diluted in water ( 1 teaspoon in $1 / 2$ liter of water) and used as natural herbal shampoo after sieving with muslin cloth locally called "Phadi". Herbal hair lotion from the crushed amla fruit (Emblicaofficinalis) and fresh lime (Citrus aurantifolia) peel which was soaked for overnight was also used as hair lotion to wash the hair after washing with artificial shampoo. Then it is washed with fresh water again. Such types of hair lotion 
Table 7: Plants used for skin and hair care [4-6,9,12-19]

\begin{tabular}{|c|c|c|}
\hline Scientific name (family) & Vernacular name & Parts used \\
\hline \multicolumn{3}{|l|}{ Emolient } \\
\hline Polygonum perfoliatum & Lihhar & Whole plant \\
\hline \multicolumn{3}{|l|}{ Hair supplements } \\
\hline $\begin{array}{l}\text { Agerathumconyzoides } \\
\text { (Asteraceae) }\end{array}$ & Khong-jai-napi & Leaves \\
\hline $\begin{array}{l}\text { Cymbopoganflexosus } \\
\text { (Poaceae) }\end{array}$ & Houna & Leaves \\
\hline Citrus laltipes (Rutaceae) & Hei-ribob & Fruit \\
\hline $\begin{array}{l}\text { Eupatorium odoratum } \\
\text { (Asteraceae) }\end{array}$ & Hanurei & Leaves \\
\hline $\begin{array}{l}\text { Eucalyptus citriodora } \\
\text { (Myrataceae) }\end{array}$ & Nasik & Leaves \\
\hline $\begin{array}{l}\text { Pogostemonbengalensis } \\
\text { (Lamiaceae) }\end{array}$ & Lamthoiding & Leaves, root \\
\hline $\begin{array}{l}\text { Pogostemonparviflorus } \\
\text { (Asteraceae) }\end{array}$ & Sangbrei & Whole plant \\
\hline $\begin{array}{l}\text { Spondiaspinnata } \\
\text { (Anacardiaceae) }\end{array}$ & Heining & Fruit, leaves \\
\hline $\begin{array}{l}\text { Kaempferia galangal } \\
\text { (Zingiberaceae) }\end{array}$ & Yaithamnamanbi & Rhizome \\
\hline Rhussemialata (Anacardiaceae) & Heimang & Leaves, fruit \\
\hline Allium odorum (Alliaceae) & Maroinakupi & Leaves \\
\hline Oxalis corniculata & Yensil & Whole plant \\
\hline \multicolumn{3}{|c|}{ Components in traditional shampoo preperation } \\
\hline $\begin{array}{l}\text { Emblica officinalis } \\
\text { (Euphorbiaceae) }\end{array}$ & Heikru & Fruit \\
\hline Glycomisarborea (Rutaceae) & Yong komla & Leaves \\
\hline Centelaasiatica (Apiaceae) & Peruk & Whole plant \\
\hline $\begin{array}{l}\text { Hibiscus rosachinensis } \\
\text { (Malvaceae) }\end{array}$ & Juba-kushum & $\begin{array}{l}\text { Leaves, } \\
\text { flower }\end{array}$ \\
\hline $\begin{array}{l}\text { Xylosmalongifolia } \\
\text { (Flacourtiaceae) }\end{array}$ & Nong-leishang & Leaves \\
\hline Eucalyptus globolus (Malvacea) & Nasik & Leaves \\
\hline $\begin{array}{l}\text { Ageratum conyzoides } \\
\text { (Asteraceae) }\end{array}$ & Khonggainapi & $\begin{array}{l}\text { Tender } \\
\text { shoot, leaves }\end{array}$ \\
\hline $\begin{array}{l}\text { Artabotryshexapetalus } \\
\text { (Annonaceae) }\end{array}$ & Chini-champa & $\begin{array}{l}\text { Leaves, } \\
\text { flower }\end{array}$ \\
\hline $\begin{array}{l}\text { Spondiaspinnata } \\
\text { (Annacardaceae) }\end{array}$ & Heining & Leaves, fruit \\
\hline Anisomelesindica (Lamiaceae) & Thoidindagouba & Leaves \\
\hline $\begin{array}{l}\text { Hydrocotylesibthorpoides } \\
\text { (Apiaceae) }\end{array}$ & Lei peruk & Whole plant \\
\hline $\begin{array}{l}\text { Pogostemonparviflorus } \\
\text { (Laminaceae) }\end{array}$ & Sang-brei & Tender shoot \\
\hline Citrus limonia (Rutaceae) & Heijang & $\begin{array}{l}\text { Leaves, fruit } \\
\text { peel }\end{array}$ \\
\hline Citrus aurantifolia (Rutaceae) & Champra & $\begin{array}{l}\text { Leaves, fruit } \\
\text { peel }\end{array}$ \\
\hline $\begin{array}{l}\text { Magloniahodgsonii } \\
\text { (Maglonaceae) }\end{array}$ & U-thambalagangba & Flower \\
\hline Citrus sinensis (Rutaceae) & Komla & Fruit peel \\
\hline Tegeteserecta (Asteraceae) & Sanareiathonba & Leaves \\
\hline Tegetesafricana (Asteraceae) & Housanarei & Leaves \\
\hline Leucas aspera (Lamiaceae) & Mayanglembum & Leaves \\
\hline $\begin{array}{l}\text { Portulacaoleraceae } \\
\text { (Portulacaceae) }\end{array}$ & Laibakkundo & Whole plant \\
\hline Meynalaxiflora (Rubiaaceae) & Heibi & Leaves \\
\hline Artimisia vulgaris (Asteraceae) & Laibakgou & Leaves \\
\hline Vitexnegundo (Verbenaceae) & Urikshibi & $\begin{array}{l}\text { Tender } \\
\text { shoot, leaves }\end{array}$ \\
\hline Rhuschinensis (Annacardaceae) & Heimang & Leaves \\
\hline
\end{tabular}

Table 7: (Continued)

\begin{tabular}{|c|c|c|}
\hline Scientific name (family) & Vernacular name & Parts used \\
\hline Acanthus mollis (Acanthaceae) & Krishna khumbam & Leaves \\
\hline Oxalis debilis (Oxalidaceae) & Inkholyensil & Whole plant \\
\hline Citrus maxima (Rutaceae) & Nobab & Leaves \\
\hline Callistemon citrinus (Myrtaceae) & Balab lei & Shoot \\
\hline Hibiscus subdariffa (Malvaceae) & Silotsougri & Leaves \\
\hline $\begin{array}{l}\text { Mussaendaroxburghii } \\
\text { (Rubiaceae) }\end{array}$ & Hanu-rei & Leaves \\
\hline Acoruscalamus (Aeraceae) & O-hidak & $\begin{array}{l}\text { Tender } \\
\text { leaves }\end{array}$ \\
\hline Citrus reticulate (Rutaceae) & Komla heithum & Fruit peel \\
\hline $\begin{array}{l}\text { Bacopa monneiri } \\
\text { (Scrophularaceae) }\end{array}$ & Laibakkundomacha & Leaves \\
\hline Artemisia maritime (Asteraceae) & Ching laibakgou & Leaves \\
\hline Oxalis corniculata (Oxalisaceae) & Lam yensil & Whole plant \\
\hline
\end{tabular}

give a cool effect to the head and softness of hair and also prevent grayness of hair.

\section{CONCLUSION}

Unfortunately, absence of proper documentation of the traditional knowledge leads to its less understanding and is likely to be lost when it is not passed on to the next generation.Most of the systematic studies in this topic focus only on epidemiological cross sectional data analysis. True clinical trials as well as scientific laboratory based examinations to decipher the contents of the herbs were not carried out effectively. Hence, along with the basic preservation of the traditional knowledge, it is very important to find out the true scientific facts behind its efficacy, so that proper dosing and route of administration can be formulated. The medicines may then be made available beyond this region, which is very much needed in the current era of emerging resistance to common drugs.

\section{REFERENCES}

1. Singh T, Devi AR, Sharma HR, Sharma HM. Medicinal plants used in the treatment of various skin diseases by the scheduled caste community of Andro village in Imphal east district, Manipur (India). Int Scien J. 2015;2:11-9.

2. Jain V, Verma SK. Assessment of credibility of some folk medicinal claims on Bombax ceiba L. Indian J Tradit Knowl. 2014;13:87-94.

3. Athokpam R, Bawari M, Choudary MD. A review on medicinal plants of Manipur with special reference to hepatoprotection. Int J Adv Pharm Res. 2014;5:182-91.

4. Devi I, Devi U, Singh EJ. Wild medicinal plants in the hill of Manipur, India: a traditional therapeutic potential. Int J Scien Res Publicat. 2015;6:1-9.

5. Leishangthem S, Sharma LD. Study of some important medicinal plants found in Imphal-East district, Manipur, India. Int J Scien Res Publicat. 2014;4:631-6.

6. Singh V, Shah N, Rana DK. Medicinal importance of unexploited vegetable under North Eastern regions of India. J Med Plants Stud. 
2015;3:33-6.

7. Devi LR, Das AK. Study on the medicinal plants used for dermatological healthcare management practices by the Paite tribe of Manipur, India. Int J Innovat Res Scien Technol. 2015;1:192-6.

8. Saha P, Talukar AD, Ningthoujam SS, Choudary MD, Nath D, Nahar L, et al. Chemical composition, antimicrobial and antioxidant properties of seed oil plants of North East India: a review. TANG (Human Med). 2015;1:1-17.

9. Usharani L, Singh WR, Surodhani S. An ethnomedicinal plant- a less known spices used by Meitei community of Manipur. Asian J Plant Scien Res. 2015;5:84-7.

10. Barua CC, Bora M, Saikia B, Hazarika M, Misri J, Chandrabarua I. Nutritional evaluation of few selected medicinal plants of North Eastern region. Int J Pharm Bio Scien. 2015;6:538-46.

11. Ningombam DS, Singh PK. Ethnobotanical study of phologacanthusthysiformis Nees: a conserved medicinal plant of Manipur, Northeast India. Int J Herbal Med. 2014;1:10-4.

12. Singh SR, Phurailatpam AK, Senjam P. Identification of the plants use as natural herbal shampoo in Manipur. Afr J Trad Compliment Altern Med. 2014;11:135-42.

13. Nongemaithem R, Das AK. Less known ethnobotanical uses of Maring tribe of Manipur. Int J Innovat Res Scien Technol. 2015;1:142-3.

14. Devi OA, Das M, Saikia A, Das P, Sharma D. Evaluation of total mineral, calcium, selenium, iron content of ten medicinal plant extracts of Manipur having anti-inflammatory properties. J Med Plants Stud. 2016;4:189-94.
15. Barua CC, Bora M, Saikia B, Hazarika M, Misri J, Chandrabarua I. Quantitative analysis of proximate and mineral composition of a few important medicinal plants of North East region. Int J Appl Biol Pharm Technol. 2015;6:188-93.

16. Singh SL, Moirangthem N, Singh SS. Medicinal plants in Manipur: a survey. Int J Pharm Scien. 2015;5:931-7.

17. Prakash N, Ansari M A, Punita P, Sharma PK. Indigenous traditional knowledge and usage of folk bio-medicines among Rongmei tribe of Tamenglong district of Manipur, India. Afr J Tradit Complement Altern Med. 2014;11(3):239-47

18. Devi WR, Singh SBS, Singh CB. Antioxidant and anti-dermatophytic properties leaf and stem bark of Xylosmalongifoliumclos. BMC Complement Altern Med. 2013;13:1-9.

19. Moirangthem DS, Talukdar NC, Bora U, Kasoju N, Das RK. Differential effects of Oroxylumindicumbark extracts: antioxidant, antimicrobial, cytotoxic and apoptotic study. Cytotechnology. 2013;65:83-95.

20. Geng A, Dufrensne RG. Complementary and alternative medicine. Textbook of Dermatology, Bolognia JL, Jorizzo JL, Rapini RP as chief editors. $2^{\text {nd }}$ edition, MOSBYElsvier, Spain, 2043-52.

Copyright by Bishurul Hafi, et al. This is an open-access article distributed under the terms of the Creative Commons Attribution License, which permits unrestricted use, distribution, and reproduction in any medium, provided the original author and source are credited.

Source of Support: Nil, Conflict of Interest: None declared. 